Check for updates

Cite this: Analyst, 2018, 143, 4674

\title{
Estimating and correcting interference fringes in infrared spectra in infrared hyperspectral imaging
}

\author{
Ghazal Azarfar, (iD) a Ebrahim Aboualizadeh, (iD ${ }^{\mathrm{b}}$ Nicholas M. Walter, (iD ${ }^{\mathrm{b}}$ \\ Simona Ratti, ${ }^{\mathrm{C}}$ Camilla Olivieri, ${ }^{\mathrm{C}}$ Alessandra Norici, ${ }^{\mathrm{C}}$ Michael Nasse, ${ }^{\mathrm{d}}$ Achim Kohler, \\ Mario Giordano (iD c and Carol J. Hirschmugl (iD *a,b
}

\begin{abstract}
Short-term acclimation response of individual cells of Thalassiosira weissflogii was monitored by Synchrotron FTIR imaging over the span of 75 minutes. The cells, collected from batch cultures, were maintained in a constant flow of medium, at an irradiance of $120 \mu \mathrm{mol} \mathrm{m} \mathrm{m}^{-2} \mathrm{~s}^{-1}$ and at $20{ }^{\circ} \mathrm{C}$. Multiple internal reflections due to the micro fluidic channel were modeled, and showed that fringes are additive sinusoids to the pure absorption of the other components of the system. Preprocessing of the hyperspectral cube $(x, y, \operatorname{Abs}(\lambda))$ included removing spectral fringe using an EMSC approach. Principal component analysis of the time series of hyperspectral cubes showed macromolecular pool variations (carbohydrates, lipids and DNA/RNA) of less than $2 \%$ after fringe correction.
\end{abstract}

Received 16th January 2018,
Accepted 27th June 2018

DOI: $10.1039 /$ c8an00093j

rsc.li/analyst between the requirement to maintain the cells in a physiological state and the need to restrict the impact of water absorption on the biochemical signal. However prior studies ${ }^{12-15}$ revealed free flotation and mobility of the cell in this geometry, requiring rapid measurements, that reduces spectral resolution and signal to noise ratios $(\mathrm{S} / \mathrm{N})$. For example, microchannels with wedge shaped windows, that were originally designed for measuring absorbent liquids,${ }^{15}$ have been modified to provide a controlled environment for live cell measurement in transmission mode. ${ }^{16,17}$ However, not all microscope geometries allow for such microfluidic chambers, such as high numerical aperture, small working distance objectives that require thin cross section microfluidic chambers. One such chamber consists of submicrometer thick diamond windows that are deposited on Si wafers. ${ }^{18}$ When the windows of microfluidic channel are separated by a distance close to the wavelength of probing radiation, multiple internal reflections are common, and lead to well understood spectral features (sinusoidal fringes) that hinder the analysis of the data. ${ }^{19}$ These sinusoidal spectral features are called fringes, and can impact the data analysis, when their amplitude is comparable to the desired spectral features.

There are two categories of fringe correction techniques: software based and hardware based. In hardware based techniques, the sample is rotated so that the angle of incidence is equal to the Brewster angle in order to achieve maximum transmittance. ${ }^{20-22} \mathrm{~A}$ spectrum measured by this technique is only accurate in low absorptivity regions, and this experimental geometry is not always feasible, such as with high magnification high numerical aperture objectives that require a wide range of incidence angles. 
Various software-based techniques for fringe correction can be used to minimize the disturbance of biologically relevant information by spectral fringes generated by water absorption. Among these, interferogram editing, fringe fitting and subtraction from the spectrum, ${ }^{23-26}$ low frequency filtering, ${ }^{27,28}$ synthetic background generation based on the fringes in a sample interferogram, ${ }^{29}$ and digital filtering of the spectrum along with Savitzky-Golay $^{30}$ algorithm. These methods, however, are limited to special cases and cannot always be applied effectively.

We propose an innovative approach to this problem which employs combination of sub-micron thick diamond windows ${ }^{18}$ and a bright synchrotron source. ${ }^{8,9}$ For live cell monitoring with high signal to noise and diffraction limited spatial resolution a Schwarzschild objective with high numerical aperture and small working distance is employed.

Single Thalassiosira weissflogii cells were monitored over the span of 2 hours to collect high quality hyperspectral cubes containing $64 \times 64 \times 1037 \times 8(x \times y \times \lambda \times t)$ pieces of information covering spatial, spectral and temporal domains. Marine diatoms are responsible for up to $20 \%$ of global $\mathrm{CO}_{2}$ fixation. Their photosynthetic efficiency is enhanced by concentrating $\mathrm{CO}_{2}$ around Rubisco, diminishing photorespiration, but the mechanism is yet to be resolved. ${ }^{31}$ Thalassiosira weissflogii is a species of centric diatoms, a unicellular microalgae and a congeneric of a model organism, Thalassiosira pseudonana. Thalassiosira weissflogii has an average size compare to other algal cells, and it is thin enough that infrared light can transmit through it.

Spectral and spatial fringes are observed in the raw measurements, and dominate principal components. Here, a preprocessing approach including fringe correction is proposed for live single cell synchrotron FTIR imaging, and subsequent data analysis is briefly demonstrated. The introduced fringe correction method is fast, flexible and can be adjusted with respect to the geometry of the sample.

\section{Methods}

\subsection{Experiment}

Batch cultures of the marine diatom Thalassiosira weissflogii were acclimated to ambient (390 ppm) $\mathrm{CO}_{2}$, and $30 \mu \mathrm{M} \mathrm{NO}_{3}{ }^{-}$, under constant irradiance of photosynthetically active radiation (PAR), at $20^{\circ} \mathrm{C}$. The algal cells were harvested by an automatic pipette and transferred to a microfluidic channel ${ }^{18}$ that supported a controlled aqueous environment with the same conditions as in the original cultures. Measurements were conducted on three independent biological replicates (i.e. three distinct cultures).

A synchrotron based source (Infrared Environmental Imaging at the Synchrotron Radiation Center) coupled to a FTIR Bruker Hyperion 3000 microspectrometer with a focal plane array(FPA) detector $(64 \times 64 \text { pixels })^{8,9}$ was used to collect spectrally resolved images of the algal samples. A $20 \times(0.6 \mathrm{NA})$ condenser objective was used to illuminate the sample, and a $74 \times$ objective ( $0.6 \mathrm{NA}$ ) was used to collect and image the transmitted radiation onto the detector, where a geometrical sample of $0.54 \times 0.54 \mu \mathrm{m}^{2}$ per pixel was obtained. The images were collected every 15 minutes for at least 2-hours. Individual algal cells were monitored across the spectral range of $850 \mathrm{~cm}^{-1}$ to $3850 \mathrm{~cm}^{-1}$ with a spectral resolution of $4 \mathrm{~cm}^{-1}$. However the spectra were truncated to $948 \mathrm{~cm}^{-1}$ to $2946 \mathrm{~cm}^{-1}$ due to total absorption of water. The spectra were smoothed using a Savitzky-Golay function of order 2 with 9 points.

Infrared transmission hyperspectral data cubes were obtained by collecting two sets of images, one reference or background hyperspectral data cube, and one sample data cube. Reference data cubes were taken from a location as close as possible to the algal cell being studied. The second set of data cubes (sample data cubes) were taken from a region including the algal cell. The division of a sample data cube by a reference data cube allowed to remove the background effects and resulted in a transmittance datacube. Obviously, since the reference image must be devoid of algal cells, it is not possible to select the same location for both the sample and reference. The difference between the two locations can (and typically does) lead to slightly different water thicknesses, generating spatial variations and spectral fringes with multiple frequencies.

\subsection{Simulating fringes in IR transmittance spectra obtained with the micro fluidic channel}

The matrix theory of optical systems ${ }^{32}$ was used to study fringe behavior in a micro fluidic channel. In this theory, the complex amplitude of the forward and backward waves through the boundaries of an optical system is calculated by use of matrix methods. ${ }^{33}$ The wave transfer matrix of a system relates the forward and backward collected waves at input and output planes of the system. The forward and backward waves collected at the input plane of the system are shown by $U_{i}^{(+)}$, and $U_{i}^{(-)}$, while $U_{o}^{(+)}$and $U_{o}^{(-)}$denote the forward and backward waves collected at the output plane. The wave-transfer matrix $M$, relates these set of forward and backward waves as follows:

$$
\left[\begin{array}{c}
U_{o}^{(+)} \\
U_{o}^{(-)}
\end{array}\right]=\left[\begin{array}{ll}
M_{11} & M_{12} \\
M_{21} & M_{22}
\end{array}\right]\left[\begin{array}{c}
U_{i}^{(+)} \\
U_{i}^{(-)}
\end{array}\right] .
$$

The wave transfer matrix of a multilayered medium, made of $n$ layers is described by the matrix product of each single element. If the transmission matrix of each element is $M_{1}, M_{2}$, $M_{3}, \ldots M_{n}$, then the total transfer matrix is defined as:

$$
M=M_{n} \ldots M_{2} M_{1}
$$

and the transmittance through the system is:

$$
|t|=\frac{1}{M_{11}} .
$$

By solving the Maxwell's equations and applying suitable boundary conditions, the transfer matrix through a single dielectric boundary as shown in Fig. 1(a), with a refractive indexes of $n_{1}$, and $n_{2}$, is calculated as: ${ }^{33}$

$$
M=\frac{1}{2 n_{2}}\left[\begin{array}{ll}
n_{2}+n_{1} & n_{2}-n_{1} \\
n_{2}-n_{1} & n_{2}+n_{1}
\end{array}\right] .
$$


(a)

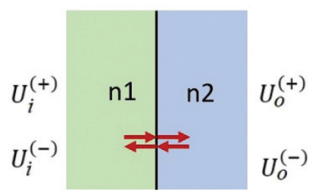

(b)

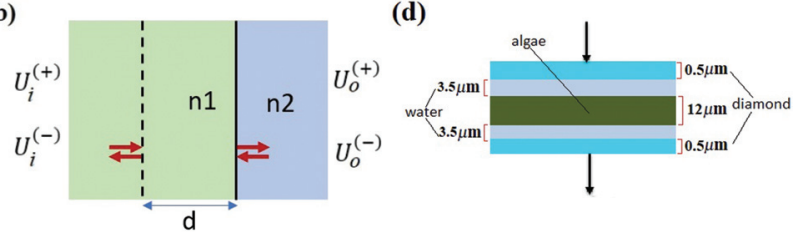

Fig. 1 (a) Single dielectric boundary made of two materials with refractive indexes of $n_{1}$, and $n_{2}$. (b) A slab of material with refractive index of $n_{1}$ and width of $d$ followed by a boundary. (c) Reference model made of diamond window and water layers. (d) Sample model made of diamond, algae and water layers.

With the same approach, the transfer matrix through a homogeneous medium with a width of $d$, followed by a boundary as shown in Fig. 1(b) is described by: ${ }^{33}$

$$
M=\frac{1}{2 n_{2}}\left[\begin{array}{ll}
\left(n_{2}+n_{1}\right) \exp (-j \phi) & \left(n_{2}-n_{1}\right) \exp (j \phi) \\
\left(n_{2}-n_{1}\right) \exp (-j \phi) & \left(n_{2}+n_{1}\right) \exp (j \phi)
\end{array}\right]
$$

where $\phi=n_{1} k_{\mathrm{o}} d$, and $k_{\mathrm{o}}$ is the propagation constant. The reference transmittance was modeled as three layers of material, including a $0.5 \mu \mathrm{m}$ diamond, a $19 \mu \mathrm{m}$ layer of water and another $0.5 \mu \mathrm{m}$ layer of diamond suspended in air as shown in Fig. 1(c). The first boundary between air and diamond was modeled using eqn (4), and the rest was modeled by means of eqn (5). The sample transmittance in the micro channel is modeled as five layers of thin film as shown in Fig. 1(d), $0.5 \mu \mathrm{m}$ layer constituted by the top diamond window, represented by the $3.5 \mu \mathrm{m}$ water layer over the cell, a $12 \mu \mathrm{m}$ layer given by the algae, a $3.5 \mu \mathrm{m}$ layer of water under the cell, and another $0.5 \mu \mathrm{m}$ layer given by the lower diamond window. The refractive index of diamond(2.4) and air(1) were considered as constant values across the entire spectra. Algal cell and water were assumed as absorbing materials with complex refractive indexes, which were calculated by the method described in ref. 26 using a standard spectrum and Kramers-Kronig relation.

The water infrared spectrum was extracted from the KnowItAll spectral collection, and the algal cell standard was estimated by the method explained in the Appendix. The absorbance is defined, starting from transmittance:

$$
A=-\log \left(\frac{T_{\text {ref }}}{T_{\text {samp }}}\right)=\log \left(T_{\text {samp }}\right)-\log \left(T_{\text {ref }}\right)
$$

where $A$ is absorbance, $T_{\text {ref }}$ is the reference transmittance, and $T_{\text {samp }}$ is the sample transmittance.

\subsection{Fringe correction}

Fringes were removed with an Extended Multiplicative Signal Correction (EMSC) method. ${ }^{34}$ EMSC is a model-based preprocessing technique that is extensively used for preprocessing of IR microspectroscopy data; it is based on Lambert-Beer law on the vibrational absorbance of materials. ${ }^{35}$ It models the absorption spectrum of a sample as the sum of its theoretical components. The EMSC model that is used to remove complex fringe patterns from spectra is the extension of the model presented by Konevskikh et al., 2015 for thin films. ${ }^{26}$ and is defined as follows:

$$
\begin{aligned}
A(\nu)= & a+b \times m(\nu) \\
& +\sum_{i}\left[d_{i} \cos \left(x_{i} \nu\right)+d_{i+1} \sin \left(x_{i} \nu\right)\right]+e \times \nu+\varepsilon(\nu)
\end{aligned}
$$

where $A$ is the absorption spectrum; $\nu$ is the wavenumber; $m(\nu)$ is the standard spectrum, $b$ is the scaling factor, $e \times \nu$ represent the linear effects, and $\varepsilon(\nu)$ is the noise combined with the spectral structures that are not accounted for by the model. One period of the fringe in the wavenumber domain is $2 \pi / x$. The $b$ parameter is not used for scaling of the data, because the thickness of the algal cell is not constant across the cell. Eqn (7) could be written in the matrix format as

$$
A=M_{\mathrm{sc}} p+E
$$

where $p$ is the fitting parameter vector, containing $a, b, d_{i}, d_{i+1}$ and $e$, which is found by least-squares regression of each spectrum onto $M_{\mathrm{sc}} . M_{\mathrm{sc}}$ is the matrix of model spectra and is defined as:

$$
M_{\mathrm{sc}}=\left[\begin{array}{cccccc}
1 & m\left(\nu_{1}\right) & \sin \left(x_{1} \nu_{1}\right) & \cos \left(x_{1} \nu_{1}\right) & \ldots & \nu_{1} \\
1 & m\left(\nu_{2}\right) & \sin \left(x_{1} \nu_{2}\right) & \cos \left(x_{1} \nu_{2}\right) & \ldots & \nu_{2} \\
\vdots & \vdots & \vdots & \vdots & \vdots & \vdots \\
1 & m\left(\nu_{k}\right) & \sin \left(x_{1} \nu_{k}\right) & \cos \left(x_{1} \nu_{k}\right) & \ldots & \nu_{k}
\end{array}\right]
$$

where $k$ is the number of wavenumbers. The standard spectrum, $m(\nu)$, is being approximated in 5 steps, as discussed in supplemental materials (see section 5.1 Standard spectrum).

For the case of two parallel windows, with a distance $d$, a sinusoid with a constant frequency depending on the optical parameters of the assembly is added to the spectrum. Thus:

$$
d=\frac{N}{2 n \Delta \nu}
$$

where $d$ is the thickness of the water layer, $N$ is the number of fringes in the wavenumber range of $\Delta \nu$, and $n$ is the refractive index of the water layer. The angular spatial frequency of the fringe, $\omega$ is defined as:

$$
\omega=4 \pi n d
$$

The unit of the angular spatial frequency is $\mathrm{rad} \mathrm{cm}$, in the rest of the paper this quantity is called frequency and its unit is displayed as cm for simplicity. The corresponding angular frequency of the fringe was calculated to be 0.0327 to $0.0465 \mathrm{~cm}$ using eqn (11).

To determine the fringe frequencies of each measured spectrum, the Fast Fourier transform (FFT) of absorbance spectrum for the spectral region between 1766 to $2790 \mathrm{~cm}^{-1}$ was calculated. The region 1766 to $2790 \mathrm{~cm}^{-1}$ is chosen because the spectrum in this spectral range is dominated by the fringe effect and there are no absorption bands. A short spectral 
region in the absorbance spectrum was chosen to calculate the FFT, and then used to calculate the frequencies that expand across the entire spectral range. To accurately detect the frequency components for such a short range of information, spectra are zero padded in the wavenumber domain. FFT of a limited signal expands in Fourier domain, hence it is reasonable to select many frequencies to reconstruct the fringe and expand it across the entire spectral range. For this specific experiment the first 10 dominant frequencies larger than 0.0053 are chosen as the fringe frequencies. A low frequency band limit of 0.0053 is chosen such that the columns of matrix $M_{\mathrm{sc}}$ in eqn (9) are orthogonal. Notice that for angular frequencies smaller than 0.0053 , cosine columns of matrix $M_{\mathrm{sc}}$ become $\approx 1$.

In the Result and discussion section, we show that the dispersion impact was negligible in the micro channel, and the fringe pattern estimated by inserting these frequencies into eqn (7) can be extended to the other spectral regions. To remove the fringes, the approximated fringe pattern was subtracted from the raw spectrum. This procedure was repeated until no fringes were observed across the region of 1766 to $2790 \mathrm{~cm}^{-1}$.

In summary, the fringe correction algorithm is:

(a) Read the spectrum with fringes.

(b) Choose a region of spectrum where fringes are dominant, and there is no absorption band.

(c) Guess the number of dominant fringe frequencies, in the region selected in $b$.

(d) While there is fringe in the spectrum do:

(d.1) Calculate the FFT of the spectral region chosen in 2.

(d.2) Choose dominant fringe frequencies.

(d.3) Form matrix $M_{\text {sc }}$.

(d.4) Estimate the fringe pattern across the whole spectral range by EMSC.

(d.5) Subtract the estimated fringe pattern from the spectrum.

\subsection{Chemogram image}

To investigate the spatial variation of the overall absorption of the sample, an integration under the spectral region (2947 $\mathrm{cm}^{-1}-1693 \mathrm{~cm}^{-1}$ and $1585 \mathrm{~cm}^{-1}-952 \mathrm{~cm}^{-1}$ ) was calculated for each pixel, excluding the water region. The resulting images, the so called chemograms, revealed the overall absorption bands and the fringes. An example is displayed in the first row of Fig. 4.

\subsection{Image segmentation}

Fringes dominated the spectra in the regions of the image where there were no algal cells, since there are no relevant chemical absorption bands at that point in space. We used a mask to select pixels that contained algal cell absorption signatures for further chemical analysis. Our uniform approach to select the pixels within and outside the mask for each IR image is as follows: (1) generation of the image due to the $\mathrm{Si}-\mathrm{O}$ peak at $1074 \mathrm{~cm}^{-1}$ (2) generation of the integrated image of silica region 1058 to $1089 \mathrm{~cm}^{-1}$ (3) addition of the two images, (4) use of Otsu's method ${ }^{36}$ to divide the pixels into two classes: class (1) cell and class (2) non-cell pixels.

Otsu's method is an optimum global thresholding method based on Bayes decision rule, which maximizes between class variance of $1 \mathrm{D}$ array (as described below), which is a normalized histogram of an image. ${ }^{36}$ To apply Otsu's method to a given $M \times N$ image, the intensity values, $I$, can be grouped into $L$ distinct levels to form a histogram, with $n_{i}$ the number of pixels with intensity level $i$. The probability of a pixel being assigned to intensity level $i$ is then given by $p_{i}=\frac{n_{i}}{M N}$. For a given $k \in[0, L]$, the histogram can be divided into two classes, $C_{1}$, containing the pixels with intensities $I \in\left[I_{0}, I_{\mathrm{k}}\right]$, and $C_{2}$, containing the pixels with intensities $I \in\left[I_{k+1}, I_{L-1}\right]$. The betweenclass variance of $C_{1}$ and $C_{2}$ can be maximized by finding $k$ such that the quantity $\sigma_{\mathrm{B}}{ }^{2}$ is maximized, which is defined as

$$
{\sigma_{\mathrm{B}}}^{2}(k)=\frac{\left[\mu_{G} P_{1}-\mu(k)\right]^{2}}{P_{1}(k)\left[1-P_{1}(k)\right]}
$$

where $\mu_{\mathrm{G}}$ is the average intensity of the entire image, $P_{1}(k)$ is the probability that a pixel is assigned to class $C_{1}$ and is calculated by $P_{1}(k)=\sum_{i=0}^{k} p_{i}$, and $\mu(k)$ is the average intensity up to level $k$

$$
\mu(k)=\sum_{i=0}^{k} i p_{i}
$$

When using Otsu's Method to create a mask, the output image $g(x, y)$, for a given input image $f(x, y)$, would be

$$
g(x, y)=\left\{\begin{array}{ll}
1 & \text { if } f(x, y)>k \\
0 & \text { if } \quad f(x, y) \leq k
\end{array} .\right.
$$

The threshold found by this algorithm selects $20 \%$ of the total pixels. The final mask is confirmed to be aligned with the algal cell observed in concurrently collected visible images. This approach facilitates automatic identification of all pixels within each cell at each time point.

\subsection{Principal component analysis (PCA)}

Principal Component Analysis (PCA) reduces the dimensionality of large data sets. When PCA is applied to spectral analysis (as a function of wavenumber) it facilitates further chemical analysis. Thus, the application of a PCA allows to decompose the spectra of the pixels selected from the masked data cubes into components. Since the spectra were not scaled, the first component is equivalent to an average spectrum of the entire data set being evaluated. The next components correspond to the variances from the average, and highlight the spectral features that are responsible for the chemical variations in the samples. The remaining components are dominated by noise. Approximately 5000 spectra were extracted from each individual algal cell ( 8 measurements over time span of two hours - 3 replicates) to identify the average spectrum and largest variance spatial components (PC2 and PC3). The original prepro- 
cessed spectra were fed into the MATLAB Statistics and Machine Learning Toolbox for PCA.

Only wavenumber ranges of 2946 to $2489 \mathrm{~cm}^{-1}, 2219$ to $1702 \mathrm{~cm}^{-1}$, and 1581 to $950 \mathrm{~cm}^{-1}$ were considered as PCA variables to remove the impact of water and $\mathrm{CO}_{2}$ absorption bands.

\section{Results and discussion}

There needs to be sufficient water in the micro-fluidic chamber to maintain cells alive; too much water, however, would absorb all the incident infrared radiation across the entire bandwidth. The thin windows may bow under the pressure of the water, causing the spacing between the diamond windows to vary. This is important because amplitude and frequency of multiple internal reflection at the interface of diamond/water depends on the spacing between the diamond windows; since this spacing is not constant throughout the chamber, the extent of these reflections vary from measurement to measurement and from place to place within the assembly, and hinder the quality of chemical images.

The geometry of the microfluidic channel leads to infrared images that contain both spatial variations and spectral fringes. Interpretation and statistical analysis of spectra with fringes can be misleading and even impossible. Appropriately executed fringe correction substantially improves the quality of images and spectra, and facilitates the analysis of the hyperspectral cube.
Recent work by Konevskikh et al., $2015^{26}$ has demonstrated that fringes in a thin film with nominal refractive index of less than 2.4, can be determined and removed successfully from simulated data with a single frequency sinusoidal fringes impacting the spectra. Konevskikh et $a .^{26}$ has shown the validity of the technique for a thin film of beef muscle tissue. Here, we have extended this work, modeling a micro fluidic channel as a seven layer assembly consisting of air, diamond window, water, algae, water, diamond window and air. The simulations show that dispersion effect are negligible in the micro fluidic channel, similar to Konevskikh et al., $2015^{26}$ approach. We further extended prior work to account for multiple sinusoidal frequency components. Fig. 2(a) and (b) show the complex refractive indexes of the water, and algal cell, calculated by Kramers-Kronig. Fig. 2(c) shows simulated absorbance through the micro channel, with an algal cell (sample red curve), compared to a region with no algal cell (reference blue curve). The algae absorbance spectrum (black curve) is calculated by subtracting the reference from the sample spectrum using eqn (6). Fig. 2(d) shows the final simulated algal cell spectrum. In the measured data absorption is set to zero between 1580 to $1700 \mathrm{~cm}^{-1}$, since the bending vibration of the water absorption leads to a weak signal and is dominated by noise (see section B of the Appendix). The peaks at $1075 \mathrm{~cm}^{-1}$, corresponding to $\mathrm{C}-\mathrm{O}-\mathrm{C} /$ polysaccharides and $\mathrm{Si}-\mathrm{O}$ of the silica shell, ${ }^{37}$ DNA-RNA at $1160 \mathrm{~cm}^{-1},{ }^{38} \mathrm{P}=\mathrm{O}$ at $1240 \mathrm{~cm}^{-1,37}$ the $\mathrm{N}-\mathrm{H}$ bending/Amide II for proteins at $1545 \mathrm{~cm}^{-1}, 37 \mathrm{C}=\mathrm{O}$ stretch associated with phospholipid at $1740 \mathrm{~cm}^{-1}$ (ref. 37) and $\mathrm{CH}_{2} /$ lipid at $2852 \mathrm{~cm}^{-1}$ and $2922 \mathrm{~cm}^{-1}$ (ref. 39) are present in (a)
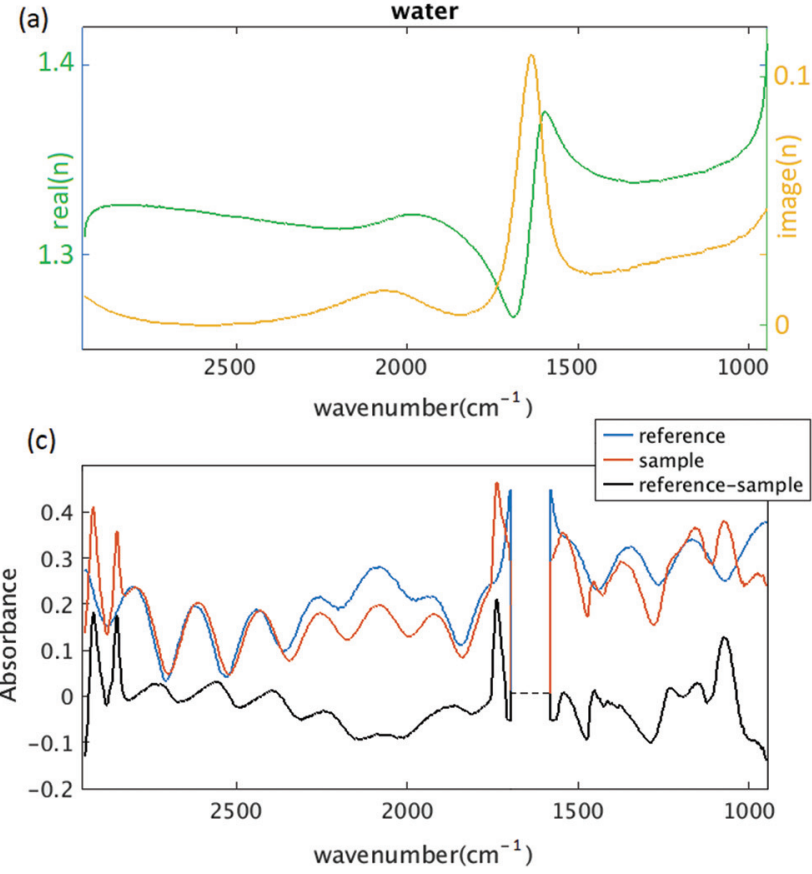

(b)
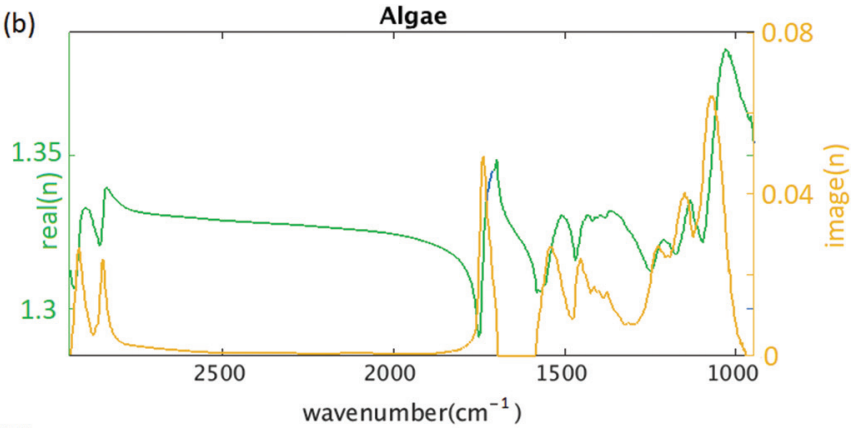

(d)

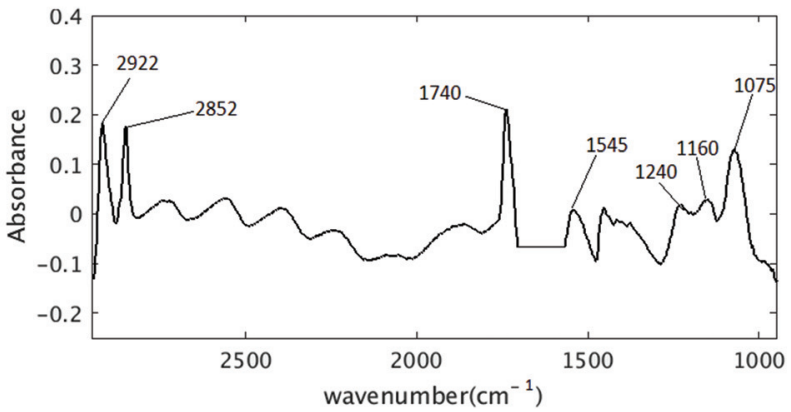

Fig. 2 Simulating the algal cell spectrum. (a) Complex refractive index of water. (b) Complex refractive index of algae. (c) The blue curve simulates the absorption through the channel, in the absence of algal cell; the red curve simulates the absorption through the channel including the algal cell (the reference transmittance is assumed to be 1 for red and blue curves). The black curve is calculated by subtracting the blue curve from the red one. (d) The simulated algal cell absorbance, the water absorption band at 1580 to $1700 \mathrm{~cm}^{-1}$ is deleted. 
the simulated data. The fringe correction algorithm applied to a simulated algal spectrum is illustrated in Fig. 3. A simulated spectrum that contains spectral fringes from multiple internal reflections is shown in Fig. 3(a). The red box shows the spectral region of 1766 to $2790 \mathrm{~cm}^{-1}$ that was dominated by fringes. The FFT of this spectral range is shown in Fig. 3(b). The first 10 dominant frequencies larger than 0.0053 were selected for fringe estimation, as discussed in section 2.3 (see section $\mathrm{C}$ of the Appendix). The fringe pattern shown in Fig. 3(c) was estimated by solving eqn (7). The approximated spectral fringe pattern was subtracted from the original spectrum, and is shown in Fig. 3(d). We reiterated this algorithm, until the corrected spectrum become free of fringes, between 1766 to $2790 \mathrm{~cm}^{-1}$, and the resulting spectrum did not change. In this case, the corrected spectrum was free of fringes after two repetitions of the algorithm. The FFT of the spectrum in the spectral range between 1766 and $2790 \mathrm{~cm}^{-1}$ after the first iteration of the fringe correction algorithm is shown in Fig. 3(e). To estimate fringes in the second iteration, the frequencies highlighted with red in Fig. 3(e) were selected, the first 10 dominant frequencies. The calculated EMSC fringe is shown in Fig. 3(d). The corrected spectrum was compared to the standard spectrum in Fig. 3(f), with a residual between the two spectra to highlight the differences. Fig. 3(f) shows that the corrected spectrum that is calculated by removing fringes with the EMSC approach is in a good agreement with the standard algae spectrum without fringe. It is well known that strong absorption bands (changes in indices of refraction) can induce dispersion in the spectral range. ${ }^{40}$ To determine the impact of the $\mathrm{OH}$ bending water absorption band on the multiple internal reflection sinusoidal fringe pattern, we inspected the residual between the corrected and standard spectra. The residual has a maximum of $15 \%$ at $1747 \mathrm{~cm}^{-1}$, and shows only noise for all frequencies. This shows dispersion is negligible. Dispersion is negligible, if the real and imaginary parts of the refractive indices of water, algae and diamond (shown in Fig. 2(a) and (b)) are less than 2.4 and 0.1 , respectively. This is consistent with the simulation results published by (a)

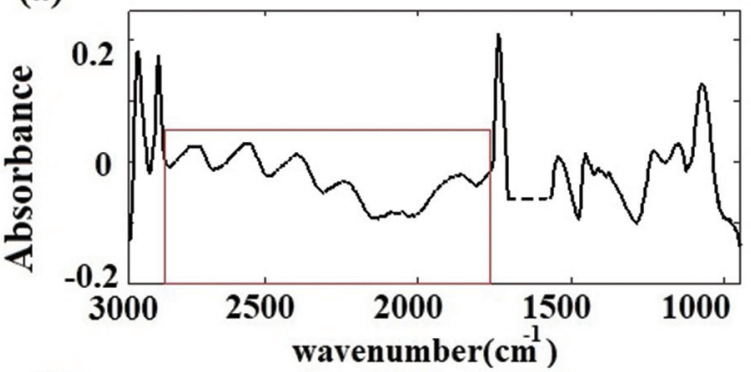

(b)

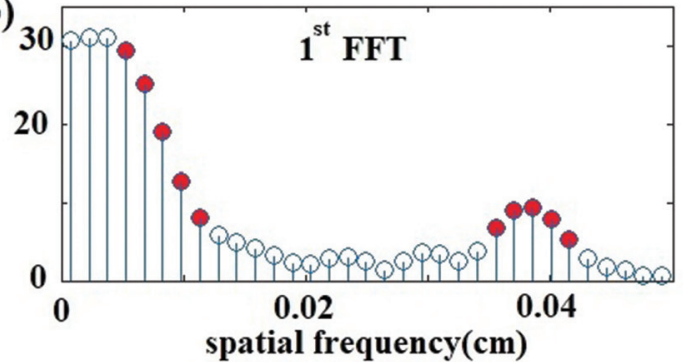

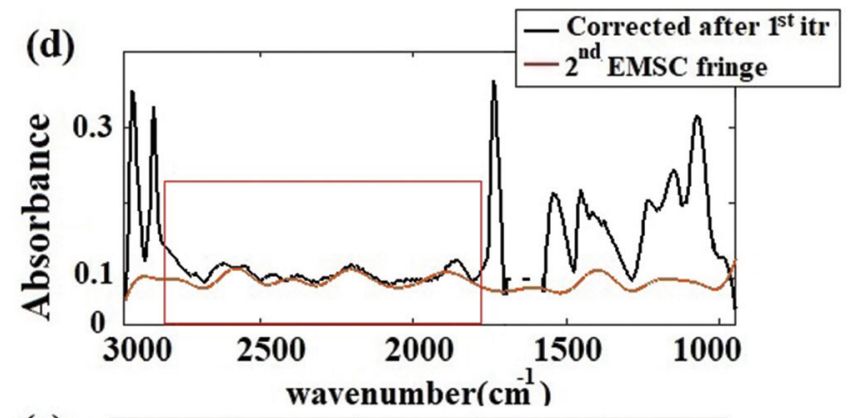

(e) 10
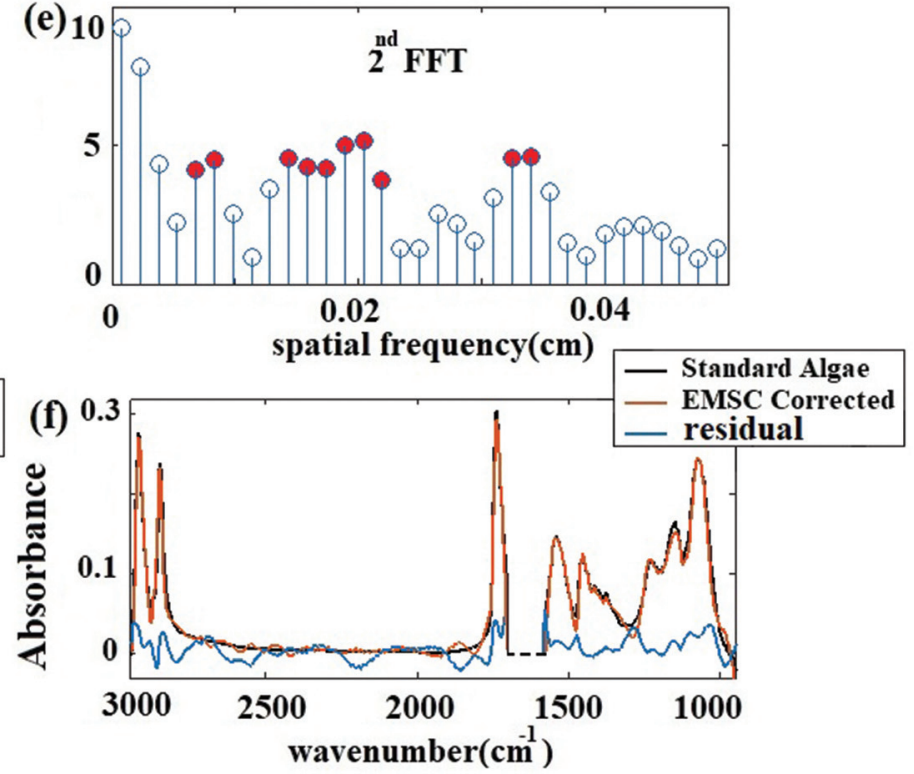

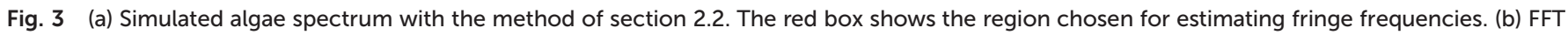

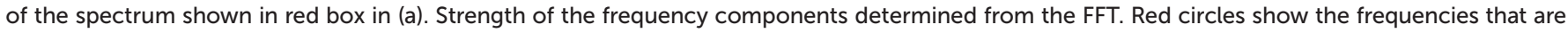

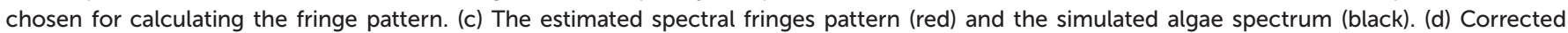

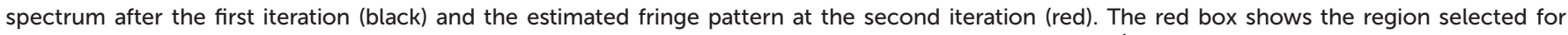

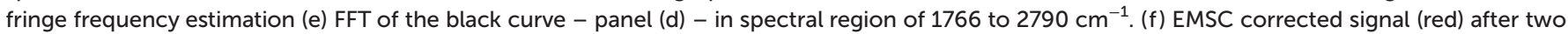
iterations compared to the standard algae spectrum (black). The residual (blue) has maximum of $15 \%$ at $1747 \mathrm{~cm}^{-1}$. 
Konveskikh et al., 2015, where there was a negligible impact on the sinusoidal fringe pattern from multiple internal reflections for real parts of indices of refraction less than 2.4. Note that the water absorption band at 1580 to $1700 \mathrm{~cm}^{-1}$ is set to zero, since it is dominated by noise in the experiment (see section B of the Appendix).

The corrected spectrum was compared to the standard spectrum in Fig. 3(f). Fig. 3(f) shows that the fringe corrected spectrum that is calculated by removing fringes with the EMSC approach is in a good agreement with the standard algae spectrum without fringe. The maximum residual of $15 \%$ was observed at $1747 \mathrm{~cm}^{-1}$. This itself is a manifestation of negligible dispersion. Small differences between the corrected and the standard algae spectrum were due to the dispersion effect $^{33}$ happening at the absorption bands. A slight variation in the imaginary part of the refractive index leads to fluctuations in the real part of the refractive index, resulting in dispersion. The algal cell, water and diamond windows are sufficiently low absorbing in the spectral range 948 to $2946 \mathrm{~cm}^{-1}$, such that the dispersion due to the absorption is negligible for the scattering of infrared radiation at the micro channel. The imaginary part of the refractive indexes of water, algae and diamond (shown in Fig. 2(a) and (b)) are less than 0.1 . The water absorption band at 1580 to $1700 \mathrm{~cm}^{-1}$ is blanked out, due to the limitation of the experiment (see section B of the Appendix). This simulation shows that, the absorbance spectra were sums of sinusoidal fringe contributions from multiple internal reflections and absorption bands from the imaginary part of the sample refraction index.
Two different cases of fringes were observed in experimental data sets. Case I: Fringes appear with a high amplitude in the average spectrum (Fig. 4 Case I). Case II: Fringes appear with a low amplitude in the average spectrum (Fig. 4 Case II). The strength of the fringe patterns in the data appears to depend on the orientation of the diamond windows in different replicates, and will be discussed further below. To model Case I (by the method described in section 2.2) the refractive index of the diamond was set to 2.4. To model Case II, the refractive index of the diamond was set to 1.5 to model the situation in which the two diamond windows were not parallel to each other due to deformation caused by the weight of water. In the case of non-parallel diamond windows, the interference effect was smaller and resulted in fringes with smaller amplitudes. A refractive index of $\mathbf{1 . 5}$ for the diamond layers was used to model the situation in which the change in the refractive index of the diamond-water interface was small. This situation was assumed for the case of non-parallel windows, since in this configuration the change in the angle of incidence would give similar optical response. For such layered systems, interfaces with similar indices of refraction resulted in smaller reflections; therefore, the fringes due to multiple internal reflections have lower amplitudes. In both cases, the EMSC estimated fringe pattern, accurately modeled the fringes, which means dispersion effect is negligible in both cases. The estimated fringes in region of 1766 to $2790 \mathrm{~cm}^{-1}$ can be extended to the whole spectral region. The maximum error occurred at the water absorption peak at $\sim 1640 \mathrm{~cm}^{-1}$, which was excluded from PCA and further analysis of the cell.

(a)
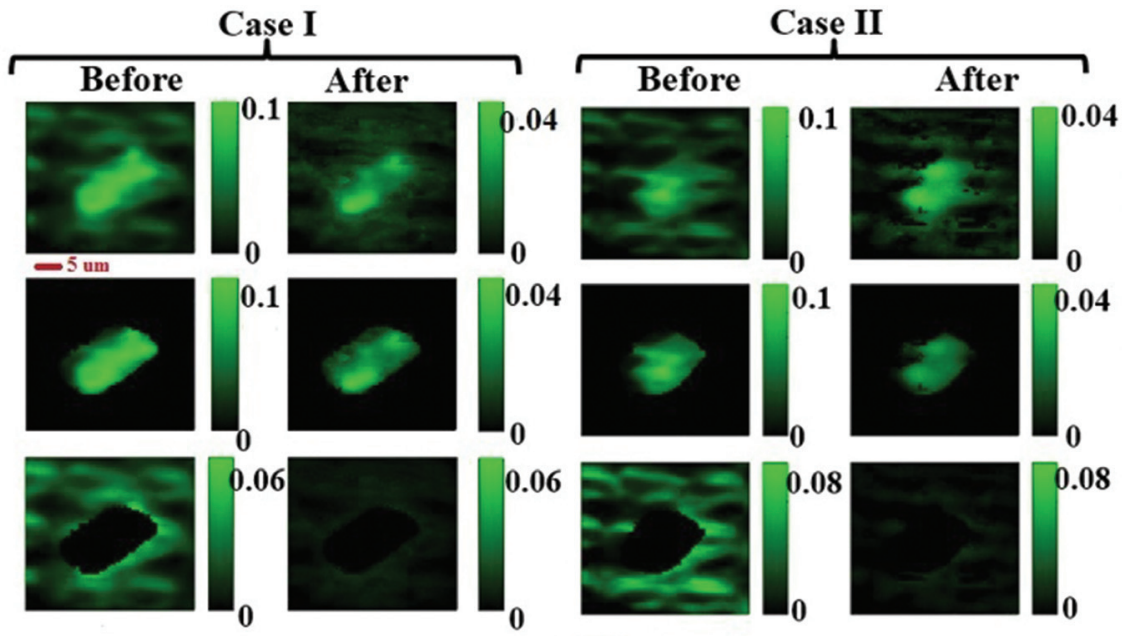

(d)
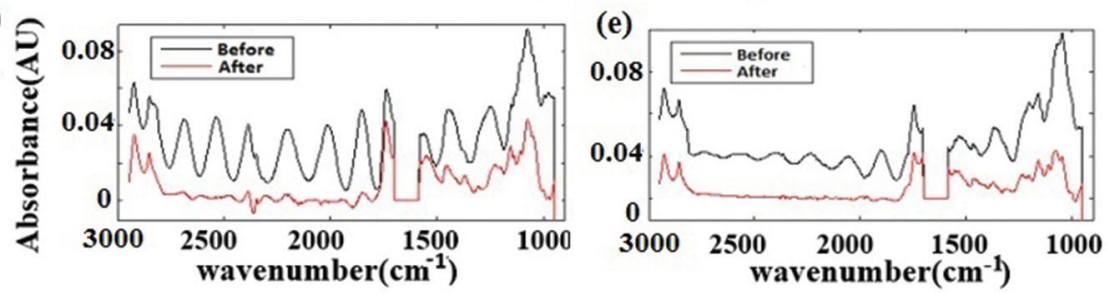

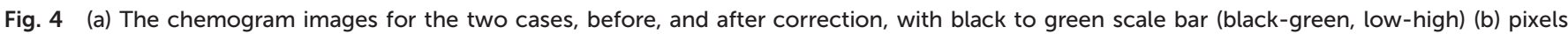
which are classified as cell by Otsu's method (c) pixels which are classified as background. (d) Average spectra before and after fringe correction. 
(a)

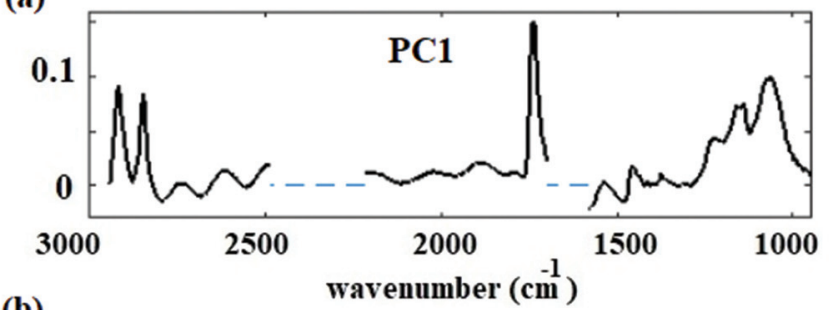

(b)

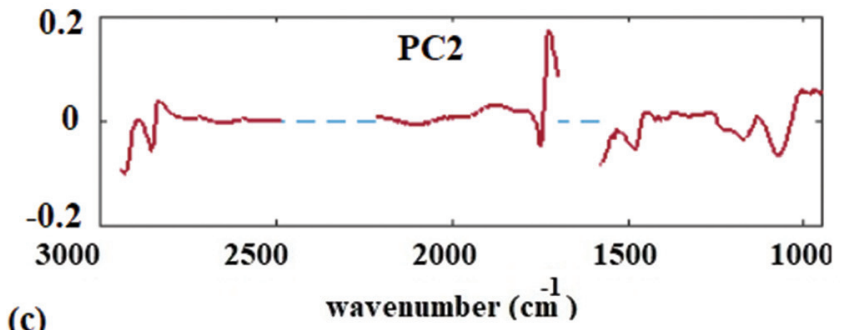

(c)

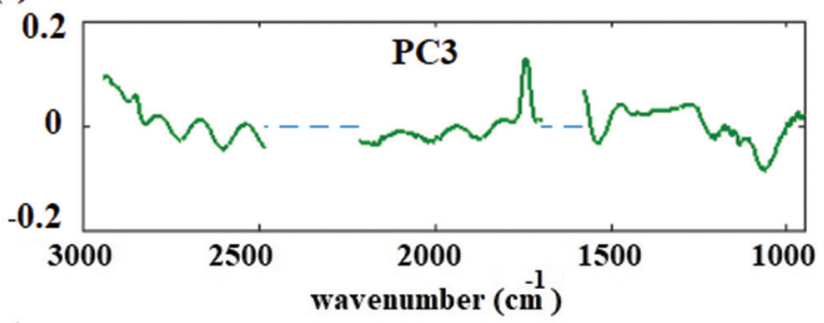

(d)

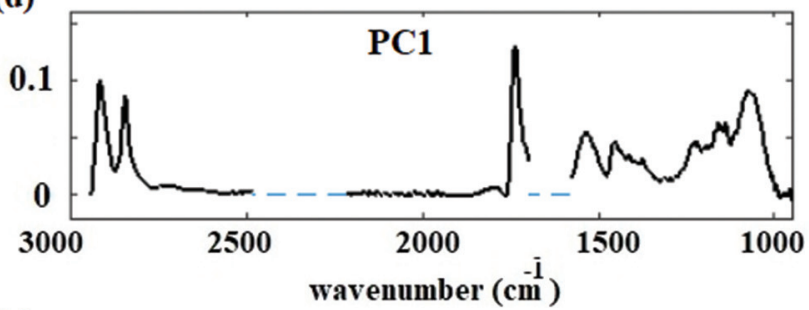

(e)

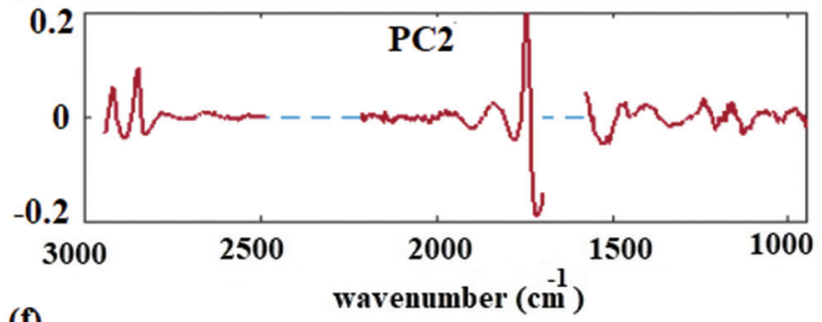

(I)

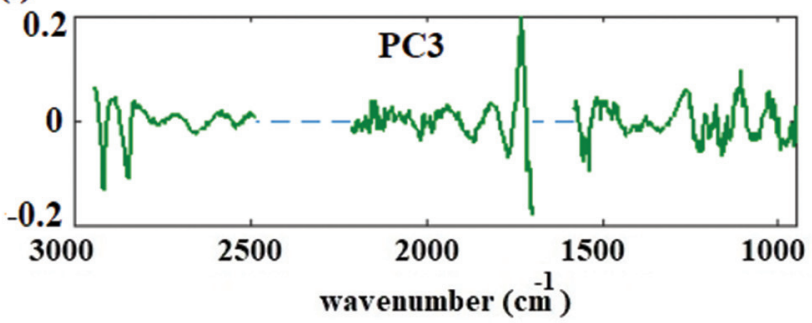

Fig. 5 Spectral principal components before and after correction. (a) First principal component before correction. (b) Second principal component before correction. (c) Third principal component before correction. (d) First principal component after correction. (e) Second principal component after correction. (f) Third principal component after correction.

Fig. 4(a) shows the measured chemogram images before and after fringe correction. The images were classified in "cell" and "non-cell" with Otsu's method. Fig. 4(b) and (c) shows pixels segregated within cells and background pixels. The average spectra before and after fringe correction (for the cell pixels) are plotted in Fig. 4(d). Note that there are smaller spectral variations and spatial fringes in the chemogram images and the average spectra after correction compare to the raw data. Importantly, fringe correction preserved strong absorbance features and even revealed originally known peaks (due to varying atmospheric $\mathrm{CO}_{2}$ in the optical beam path). Fig. 4(c) shows that the spatial background variation decreased after fringe correction in both cases, demonstrating that the spatial fringes were attenuated after spectral fringe correction.

Reducing the data to principal components allows an overall assessment of data before and after correction. The spectral results of the PCA before and after fringe correction are shown in Fig. 5. Fig. 5(a), (b) and (c) show the first, second, and third principal components of the hyperspectral cube corrected by basic EMSC model, ${ }^{41,42}$ respectively; Fig. 5(d), (e) and (f) shows the first, second, and third principal components after fringe correction. Original data variances were dominated by fringes. (Similar results were obtained for PCA of spectra with significant scattering contributions. Scattering features can dominate spectral principal components before preprocessing corrections.) Once the spectral fringes were removed for each individual spectrum, spatial variations and spectral fringes were greatly reduced in the frequency dependent loadings of principal components (average, second and third principal components). After removing the fringes and applying PCA, the frequency dependent variance spectra showed signatures corresponding to lipid and carbohydrate associated bands of the spectra Fig. 5(d)-(f). The contributions of the second and third principal components to describing the overall spectra are less than $2 \%$ of the total variation. This result suggests that changes in the lipid and carbohydrate macromolecular pools are minimal, as one would expect for a cell maintained at constant, controlled conditions, and measured over a period of several hours. ${ }^{18}$ Detecting these small variations is possible only after applying data preprocessing algorithms. The results (PC loadings) of the analysis on three replicates are consistent and prove the reliability of the procedure.

\section{Conclusions}

A single Thalassiosira weissflogii cell mounted in a micro fluid channel was monitored by FTIR imaging, under controlled condition for 75 minutes by FTIR imaging. Fringes due to multiple internal reflections of the light in the macro channel were modeled by matrix theory of multi layer media. We 
showed that the dispersion effect was negligible in this geometry. Fringes behaved as additive sinusoidal in the spectral range between 950 and $2950 \mathrm{~cm}^{-1}$, and were removed from hyperspectral cube of data by extended multiplicative signal correction. Principal component analysis of $\sim 4000$ spectra afforded significant improvement in spectral loading components after fringe correction. Our results demonstrate that lipid and carbohydrate macromolecular pools have minimal changes for the algal cell maintained at constant controlled conditions.

\section{Conflicts of interest}

There are no conflicts of interest to declare.

\section{Appendix}

\section{A. Standard spectrum}

To estimate a standard spectrum (denoted by $m$ in eqn (1)) with no fringes similar to what is presented in ref. 26 , the following steps are taken:

(1) Water absorption regions of 2946-2489 $\mathrm{cm}^{-1}$, 2219-1702 $\mathrm{cm}^{-1}$, and 1581 to $950 \mathrm{~cm}^{-1}$ are set to zero.

(2) The average spectrum of the hyperspectral data is calculated, as the base of the standard spectrum. It is considered that there is minimum fringes present in the average spectrum.

(3) Regions of $[1585,962]$ and $[2780,1766] \mathrm{cm}^{-1}$ are separately, baseline corrected.

(4) The region of 1766 to $2790 \mathrm{~cm}^{-1}$ is replaced with corresponding region of standard matrigel.

(5) The resulting spectrum is normalized at $\mathrm{Si}-\mathrm{O}$ peak at $1076 \mathrm{~cm}^{-1}$.

\section{B. Algal cell measurement}

The size of the algal cell is an important factor, chosen for several reasons. The first was to match an area that could be imaged simultaneously with the infrared camera and the microscope. The second was such that the thickness of the water/media layer to maintain the viability of the cell would be thin enough to transmit infrared photons across a majority of the infrared spectral range. Since there are strong absorption bands at $1630 \mathrm{~cm}^{-1}$ and above $3000 \mathrm{~cm}^{-1}$ for water, and a thick layer of water is known to absorb strongly across the entire infrared spectral range, limiting the thickness of the water layer combined with using a bright infrared source of light is critical to being able to detect the remaining mid-infrared signatures of the algal cell while viable.

Fig. 6 shows raw measured absorbance spectra from two distinct pixels, one from water region and the other from algal cell. In both cases, the signal is unreliable between spectral ranges of 3654 to $3100 \mathrm{~cm}^{-1}, 1700$ to $1580 \mathrm{~cm}^{-1}$, which correspond to the $\mathrm{OH}$ stretching and $\mathrm{OH}$ bending vibrations. Those spectral regions are neglected for further analysis and the

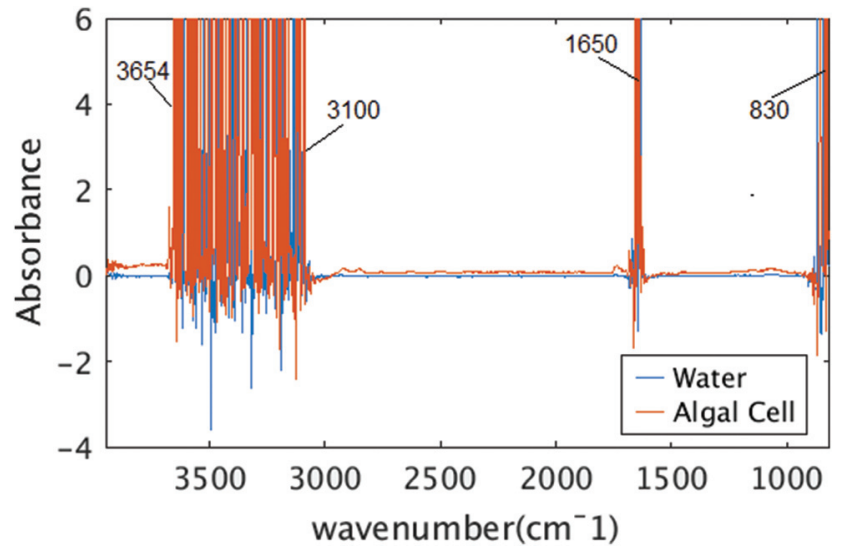

Fig. 6 Raw measured spectra. Two raw spectra in spectral range of 850 to $3950 \mathrm{~cm}^{-1}$ are shown, one from algal cell, and one from water.

experiment did not provide any information about the water absorption bands or the protein amide I bands that overlap this same frequency.

\section{Fringe frequencies}

In Fig. 3(b), the first 10 dominant fringe frequencies are chosen for fringe correction. Fig. 7 shows the result of simply including the fringes that would arise from the frequencies

(a)
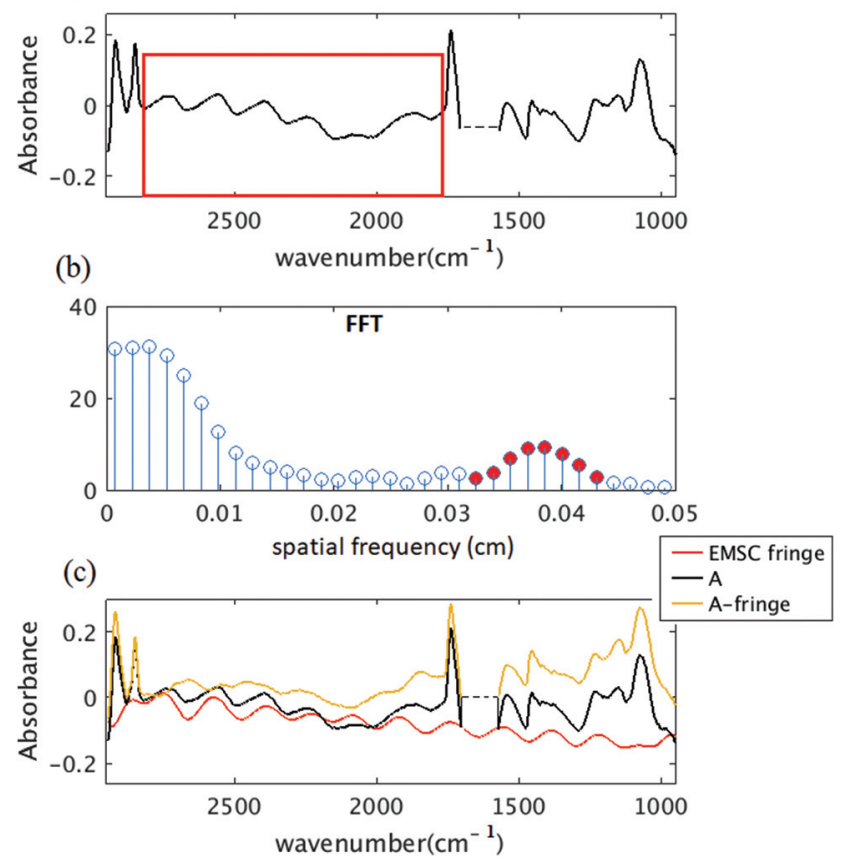

Fig. 7 (a) Simulated algal cell absorption spectrum with the method of section 2.2. The red box shows the region chosen for FFT. (b) FFT of the spectral range shown in red box shown in (a). (c) The black curve shows the simulated algal cell with fringes, the red curve shows the estimated fringe pattern using EMSC method with red frequencies of (b), the yellow curve shows the corrected spectrum (subtraction of red from black). 
$\sim 0.04 \mathrm{~cm}$ as it was suggested by Griffiths et al. (2007). ${ }^{43}$ Fig. 7(a) shows the simulated spectrum with the method of section 2.2. and the region chosen for taking the FFT. Fig. 7(b) shows the FFT of the signal, the red circles show the frequencies that are selected for calculating the fringes. Fig. 7(c) shows the simulated spectrum (black), the estimated fringe by EMS (red) using the frequencies Fig. 7(b) and the corrected spectrum (yellow brown). Clearly, the corrected spectrum is still hindered by a fringe pattern with additional frequency components. This is reasonable, since the frequencies are calculated from a FFT of a limited spectral range, many frequency components will be needed to accurately predict the correct fringe pattern due to the uncertainty principle.

\section{Acknowledgements}

The authors acknowledge UWM Physics department, NSF grants CHE-1508240 and CHE-1112433 and GAČR 16-16343S for their support.

\section{References}

1 H. Y. N. Holman, H. A. Bechtel, Z. Hao and M. C. Martin, Anal. Chem., 2010, 82, 8757-8765.

2 K. Loutherback, G. Birarda, L. Chen and H.-Y. Holman, Protein Pept. Lett., 2016, 23, 273-282.

3 L. M. Miller, Q. Wang, T. P. Telivala, R. J. Smith, A. Lanzirotti and J. Miklossy, J. Struct. Biol., 2006, 155, 3037.

4 A. Didonna, L. Vaccari, A. Bek and G. Legname, ACS Chem. Neurosci., 2011, 2, 160-174.

5 G. Grenci, G. Birarda, E. Mitri, L. Businaro, S. Pacor, L. Vaccari and M. Tormen, Microelectron. Eng., 2012, 98, 698-702.

6 E. Lipiec, G. Birarda, J. Kowalska, J. Lekki, L. Vaccari, A. Wiecheć, B. R. Wood and W. M. Kwiatek, Radiat. Phys. Chem., 2013, 93, 135-141.

7 L. Vaccari, G. Birarda, L. Businaro, S. Pacor and G. Grenci, Anal. Chem., 2012, 84, 4768-4775.

8 M. J. Nasse, M. J. Walsh, E. C. Mattson, R. Reininger, A. Kajdacsy-Balla, V. Macias, R. Bhargava and C. J. Hirschmugl, Nat. Methods, 2011, 8, 413-416.

9 C. J. Hirschmugl and K. M. Gough, Appl. Spectrosc., 2012, 66, 475-491.

10 I. Meglinski, Biophotonics for Medical Applications, 2014.

11 K. Rahmelow and W. Hübner, Appl. Spectrosc., 1997, 51, 160-170.

12 M. K. Kuimova, K. L. A. Chan and S. G. Kazarian, Appl. Spectrosc., 2009, 63, 164-171.

13 K.-I. Miyamoto, P. Yamada, R.-T. Yamaguchi, T. Muto, A. Hirano, Y. Kimura, M. Niwano and H. Isoda, Cytotechnology, 2007, 55, 143-149.
14 M. W. Peter, A. SuciaJulia and D. Vranya, Biomaterials, 1998, 19, 327-339.

15 D. M. Wieliczka, S. Weng and M. R. Querry, Appl. Opt., 1989, 28, 1714-1719.

16 H. Y. N. Holman, R. Miles, Z. Hao, E. Wozei, L. M. Anderson and H. Yang, Anal. Chem., 2009, 81, 8564-8570.

17 G. Birarda, A. Ravasio, M. Suryana, S. Maniam, H.-Y. N. Holman and G. Grenci, Lab Chip, 2016, 16, 1644-1651.

18 M. J. Nasse, S. Ratti, M. Giordano and C. J. Hirschmugl, Appl. Spectrosc., 2009, 63, 1181-1186.

19 I. Amr, P.-C. Adriana and P. Chad, Can. J. Phys., 2013, 91, 910-923.

20 P. J. Farrington, D. J. T. Hill, J. H. O'Donnell and P. J. Pomery, Appl. Spectrosc., 1990, 44, 901-903.

21 N. J. Harrick, Appl. Spectrosc., 1977, 31, 548-549.

22 E. Hecht, OPTICS, 2002.

23 T. Hirschfeld and A. W. Mantz, Appl. Spectrosc., 1976, 30, 552-553.

24 T. Hirschfeld, Appl. Opt., 1978, 17, 1400-1412.

25 F. R. S. Clark and D. J. Moffatt, Appl. Spectrosc., 1978, 32, 547-549.

26 T. Konevskikh, A. Ponossov, R. Blümel, R. Lukacs and A. Kohler, Analyst, 2015, 140, 3969-3980.

27 A. H. Lipkus, T. J. Lenk, K. K. Chittur and R. M. Gendreau, Biopolymers, 1988, 27, 1831-1838.

28 A. M. Pistorius and W. J. DeGrip, Vib. Spectrosc., 2004, 36, 89-95.

29 G. C. Mellau and B. P. Winnewisser, ASP Conference Series, Grevesse, 1995, pp. 138-139.

30 A. Savitzky and M. J. Golay, Anal. Chem., 1964, 36, 1627-1639. 31 K. Roberts, E. Granum, R. C. Leegood and J. A. Raven, Plant Physiol., 2007, 145, 230-235.

32 E. Merzbacher, Quantum Mechanics, 3rd edn, 1998.

33 B. Saleh and M. C. Teich, Fundamentals of Photonics, 2007.

34 H. Martens and E. Stark, J. Pharm. Biomed. Anal., 1991, 9, 625-635.

35 N. K. Afseth and A. Kohler, Chemom. Intell. Lab. Syst., 2012, 117, 92-99.

36 R. C. Gonzalez and R. E. Woods, Digital Image Processing.

37 Y. Meng, C. Yao, S. Xue and H. Yang, Bioresour. Technol., 2014, 151, 347-354.

38 T. Ramachandra and M. D. M. Madhab, in Proceedings of the 8th National Conference on Indian Energy Sector.

39 D. Y. Duygu, A. U. Udoh, T. B. Ozer, A. Akbulut, I. A. Erkaya, K. Yildiz and D. Guler, Afr. J. Biotechnol., 2012, 11, 3817-3824.

40 P. Bassan, A. Kohler, H. Martens, J. Lee, H. J. Byrne, P. Dumas, E. Gazi, M. Brown, N. Clarke and P. Gardner, Analyst, 2010, 135, 268-277.

41 H. Martens, J. P. Nielsen and S. B. Engelsen, Anal. Chem., 2003, 75, 394-404.

42 A. Kohler, C. Kirschner, A. Oust and H. Martens, Appl. Spectrosc., 2005, 59, 707-716.

43 P. Griffiths and J. De Haseth, Fourier Transform Infrared Spectrometry, 2007. 